\title{
Robust semidefinite programming approach to the separability problem
}

\author{
Fernando G. S. L. Brandão* and Reinaldo O. Vianna ${ }^{\dagger}$ \\ Departamento de Física, Universidade Federal de Minas Gerais, Caixa Postal 702, Belo Horizonte, MG 30.123-970, Brazil
}

(Received 4 May 2004; published 9 December 2004)

\begin{abstract}
We express the optimization of entanglement witnesses for arbitrary bipartite states in terms of a class of convex optimization problems known as robust semidefinite programs (RSDPs). We propose, using well known properties of RSDPs, several sufficient tests for separability of mixed states. Our results are then generalized to multipartite density operators.
\end{abstract}

DOI: 10.1103/PhysRevA.70.062309

PACS number(s): 03.67.Mn

\section{INTRODUCTION}

Entanglement, first noticed by Einstein, Podolsky, and Rosen [1], is at the heart of quantum mechanics. Quantum teleportation, superdense coding, and cryptography [2] are achieved only when one deals with inseparable states. Thus, the determination and quantification of entanglement in a composite quantum state is one of the most important tasks of quantum information theory. In the past years a great deal of effort have been made in order to obtain the characterization of separable bipartite mixed states [3]. A finitedimensional bipartite density operator $\rho_{A B} \in \mathcal{B}\left(H_{A} \otimes H_{B}\right)$ (the Hilbert space of bounded operators acting on $H_{A} \otimes H_{B}$ ) is separable if and only if it can be written as a convex sum of separable pure states:

$$
\rho_{A B}=\sum_{i} p_{i}\left|\psi_{i}\right\rangle_{A}\left\langle\psi_{i}|\otimes| \phi_{i}\right\rangle_{B}\left\langle\phi_{i}\right|
$$

where $\left\{p_{i}\right\}$ is a probability distribution and $\left|\psi_{i}\right\rangle_{A},\left|\phi_{i}\right\rangle_{B}$ are vectors belonging to Hilbert spaces $H_{A}$ and $H_{B}$, respectively. Despite the simplicity of this definition, no operational necessary and sufficient criterion has been found for the separability problem until now. Moreover, it was shown by Gurvits [4] that this problem is $N P$-hard. Therefore, we should not expect to find a polynomial-time algorithm which determines for any state $\rho_{A B}$, with certainty, if it is possible to decompose it in the form of Eq. (1).

A particularly useful concept is that of the entanglement witness (EW). According to [5], an operator $\rho_{A B}$ is entangled if and only if there exists a self-adjoint operator $W \in \mathcal{B}\left(H_{A}\right.$ $\left.\otimes H_{B}\right)$ which detects its entanglement, i.e., such that $\operatorname{Tr}\left(W \rho_{A B}\right)<0$ and $\operatorname{Tr}\left(W \sigma_{A B}\right) \geqslant 0$ for all $\sigma_{A B}$ separable. This condition follows from the fact that the set of separable states is convex and closed in $\mathcal{B}\left(H_{A} \otimes H_{B}\right)$. Therefore, as a conclusion of the Hahn-Banach theorem, for all entangled states there is a linear functional which separates it from this set. Unfortunately, it is not known how to construct the EW in a canonical way and in polynomial time for every entangled state. Actually, since such a method would solve the separability problem, it cannot exist as long as the strong conjecture $P \neq N P$ is true.

\footnotetext{
*Electronic address: fgslb@ufmg.br

†Electronic address: reinaldo@fisica.ufmg.br
}

In this paper, we show that the search for an EW for arbitrary mixed states is indeed $N P$-hard. We introduce, in the context of quantum information, a class of convex optimization problems known as robust semidefinite programs (RSDPs), whose NP-hardness in most cases was already proved. This family generalizes the important semidefinite programs (SDPs), which have been increasingly used in quantum-information problems [9].

The paper is organized as follows. In Sec. II we briefly recall the definition of the EW and define our concept of the optimal entanglement witness (OEW); in Sec. III we state the basic facts about robust semidefinite programs, express the optimization of the EW as a RSDP, and provide a first approximation in terms of the SDP for the problem, which yields a sufficient criterion of separability; in Sec. IV we parametrize all possible approximations of the RSDP in terms of a multiplier matrix, reducing the search space of approximation scenes; in Sec. V we generalize our results to multipartite states. Finally, in Sec. VI, we present our conclusions and suggest directions for further research.

\section{OPTIMAL ENTANGLEMENT WITNESS}

A Hermitian operator $W \in \mathcal{B}\left(H_{A} \otimes H_{B}\right)$ is an entanglement witness if [5,6] (1) ${ }_{A}\left\langle\psi\left|\otimes_{B}\langle\phi|W| \phi\rangle_{B} \otimes\right| \psi\right\rangle_{A} \geqslant 0$, for all states $|\psi\rangle \in H_{A}$ and $|\phi\rangle \in H_{B}$; (2) $W$ has at least one negative eigenvalue; (3) $\operatorname{Tr}(W)=1$. Condition 1 assures that $\operatorname{Tr}\left(W \sigma_{A B}\right)>0$ for all separable states $\sigma_{A B}$. Condition 2 implies that $\operatorname{Tr}(W P)<0$ at least for one entangled state, for example, the projector on the eigenspace associated with the negative eigenvalue. The third condition is important in order to compare different EWs.

Definition 1. A Hermitian operator $W_{\rho_{A B}}$ is an optimal EW for the density operator $\rho_{A B}$ if

$$
\operatorname{Tr}\left(W_{\rho_{A B}} \rho_{A B}\right) \leqslant \operatorname{Tr}\left(W \rho_{A B}\right)
$$

for every EW $W$.

Although the above definition of the OEW is different from the one introduced in [7], the optimal EW's of both criteria are equal. According to [7], $W$ is optimal if and only if for all $P \geqslant 0, W^{\prime}=(1+\epsilon) W-\epsilon P$ is not an EW.

\section{ROBUST SEMIDEFINITE PROGRAMS}

In this section we will express the search of an optimal EW for an arbitrary state $\rho_{A B}$ in terms of a robust semidefi- 
nite program. A semidefinite program consists of minimizing a linear objective under a linear matrix inequality (LMI) constraint, precisely,

$$
\begin{aligned}
& \text { minimize } c^{\dagger} x \text { subject to } \\
& F(x)=F_{0}+\sum_{i=1}^{m} x_{i} F_{i} \geqslant 0,
\end{aligned}
$$

where $c \in \mathcal{C}^{m}$ and the Hermitian matrices $F_{i}=F_{i}^{\dagger} \in \mathcal{C}^{n x n}$ are given and $x \in \mathcal{C}^{m}$ is the vector of optimization variables. $F(x) \geqslant 0$ means $F(x)$ is Hermitian and positive semidefinite. SDPs are global convex optimization programs and can be solved in polynomial time with interior-point algorithms [8]. For instance, if there are $m$ optimization variables and $F(x)$ is an $n \times n$ matrix, the number of operations scales with problem size as $O\left(m^{2} n^{2}\right)$. SDPs have already been used in different problems of quantum-information theory [9] and also in the separability problem [10]. An important generalization of (3) is when the data matrices $F_{i}$ are not constant, i.e., they depend on a parameter that varies within a certain subspace. This family of problems, known as robust semidefinite programs, is given by

$$
\begin{gathered}
\text { minimize } c^{\dagger} x \text { subject to } \\
F(x, \Delta)=F_{0}(\Delta)+\sum_{i=1}^{m} x_{i} F_{i}(\Delta) \geqslant 0, \quad \forall \Delta \in \mathcal{D}
\end{gathered}
$$

where $\mathcal{D}$ is a given vectorial (sub)space. Note that problem (4) is more difficult to solve than (3), since one must find an optimization vector $x$ such that $F(x, \Delta)$ is positive semidefinite to all $\Delta \in \mathcal{D}$. One often encounters SDPs in which the variables are matrices and in which the inequality depends affinely on those matrices. These problems can be readily put in the form (3) by introducing a base of Hermitian matrices to each matrix variable. However, since most optimization solvers [11] admit declaration of problems in this most general form, it is not necessary to write out the LMI explicitly as (3), but instead make clear which matrices are variables. Equality constraints involving the optimization variables can also appear in Eqs. (3) and (4) without any further computational effort. We can now enunciate the main result of this paper.

Theorem 1. A state $\rho_{A B} \in \mathcal{B}\left(H_{A} \otimes H_{B}\right)$ is entangled, i.e., cannot be decomposed as (1) if and only if the optimal value of the following RSDP is negative:

$$
\begin{gathered}
\text { minimize } \operatorname{Tr}\left(W \rho_{A B}\right) \text { subject to } \\
\sum_{i=1}^{d_{A}} \sum_{j=1}^{d_{A}} a_{i}^{*} a_{j} W_{i j} \geqslant 0, \quad \operatorname{Tr}(W)=1 \quad \text { for all } a_{i} \in \mathcal{C}
\end{gathered}
$$

where $d_{A}$ is the dimension of $H_{A}, W_{i j}={ }_{A}\langle i|W| j\rangle_{A} \in \mathcal{B}\left(H_{B}\right)$, and $|j\rangle_{A}$ is an orthonormal base of $\mathcal{H}_{A}$. If $\rho_{A B}$ is entangled, the matrix $W$ which minimizes $\operatorname{Tr}\left(W \rho_{A B}\right)$ is the OEW for $\rho_{A B}$.

Proof. First we have to show that (5) is a genuine RSDP. Note that $W_{i j}={ }_{A}\langle i|W| j\rangle_{A}$ and the objective $\operatorname{Tr}\left(W \rho_{A B}\right)$ are both linear in the matrix variable $W$. Thus, (5) can be put in the form (4), where $\mathcal{D}$ in this case is $\mathcal{C}^{d_{A}}$. We know that a state $\rho_{A B}$ is entangled if and only if there exists an entanglement witness $W$ such that $\operatorname{Tr}\left(W \rho_{A B}\right) \leqslant 0$. It is clear that if $W$ is an EW, then ${ }_{A}\langle\psi|W| \psi\rangle_{A}$ has to be semidefinite positive for all $|\psi\rangle_{A} \in H_{A}$. Conversely, if ${ }_{A}\langle\psi|W| \psi\rangle_{A} \geqslant 0$ for all $|\psi\rangle_{A} \in H_{A}$, then $W$ is an EW, i.e., $\operatorname{Tr}\left(|\psi\rangle_{A} \otimes|\psi\rangle_{B B}\langle\psi| \otimes_{A}\langle\psi| W\right)$ $=\operatorname{Tr}_{B}\left(|\psi\rangle_{B B}\left\langle\left.\psi\right|_{A}\langle\psi|W| \psi\rangle_{A}\right) \geqslant 0\right.$ for all $|\psi\rangle_{A} \in H_{A}$ and $|\psi\rangle_{B}$ $\in H_{B}$. Thus, letting $|\psi\rangle_{A}=\Sigma_{j} a_{j}|j\rangle_{A}$, where $|j\rangle_{A}$ is an orthonormal base of $\mathcal{H}_{A}$, it is straightforward to see that the optimal $W$ given by (5) is the OEW of $\rho_{A B}$. QED.

In spite of the similarity between (3) and (4), RSDPs are in general very hard optimization problems. Actually, it was proved that robust semidefinite programs like (5) are $N P$-hard [12].

Corollary 1. The determination of the OEW for an arbitrary state $\rho_{A B}$ is an $N P$-hard problem.

Although this corollary is in complete accordance with the work of Gurvits [4], it cannot be considered a different approach leading to the same results, as the $N P$-hardness of the optimization of entanglement witnesses does not rule out the existence of a polynomial-time algorithm capable of distinguishing entangled from separable states.

Since (5) is computationally intractable, it is natural to search for approximations of it in terms of SDPs, which are very efficiently solved. These relaxations of RSDPs have been intensively studied [13] in the past years and can be classified as probabilistic or deterministic. In this paper we will focus in the deterministic relaxations, where (4) is replaced by an inner convex approximation described by a linear matrix inequality constraint. This inner approximation is then used to find an upper bound to the optimal value of (4). The probabilistic approach, which yields outstanding results on the separability problem, will be reported elsewhere. As a first example of such relaxations, consider the following adaptation of [14].

Theorem 2. A density operator $\rho_{A B}$ is entangled and the optimal value of $W$ is an EW for it if the result of the following SDP is negative:

minimize $\operatorname{Tr}\left(W \rho_{A B}\right)$ subject to

(1) $W_{k k} \geqslant 0, \quad k=1,2, \ldots, d_{A}$,

(2) $\frac{1}{d_{A}-1} W_{k k} \pm \frac{\sqrt{2}}{2}\left(W_{k j}+W_{j k}\right) \geqslant 0, \quad 1 \leqslant k \neq j \leqslant d_{A}$,

(3) $\frac{1}{d_{A}-1} W_{k k} \pm \frac{\sqrt{2}}{2 i}\left(W_{k j}-W_{j k}\right) \geqslant 0, \quad 1 \leqslant k \neq j \leqslant d_{A}$.

Proof. Note that 


$$
\begin{aligned}
\sum_{k, j} a_{k}^{*} a_{j} W_{k j}= & \sum_{1 \leqslant k \leqslant j \leqslant d_{A}}\left[\frac{1}{d_{A}-1}\left|a_{k}\right|^{2} W_{k k}+a_{k}^{*} a_{j} W_{k j}+a_{j}^{*} a_{k} W_{j k}\right. \\
& \left.+\frac{1}{d_{A}-1}\left|a_{k}\right|^{2} W_{k k}\right] \geqslant 0 .
\end{aligned}
$$

Thus, a sufficient condition to (5) is

$$
M(|e\rangle)=\left[\begin{array}{cc}
\left\langle e\left|\frac{W_{k k}}{d_{A}-1}\right| e\right\rangle & \left\langle e\left|W_{k j}\right| e\right\rangle \\
\left\langle e\left|W_{j k}\right| e\right\rangle & \left\langle e\left|\frac{W_{j j}}{d_{A}-1}\right| e\right\rangle
\end{array}\right] \geqslant 0, \quad \forall|e\rangle \in \mathcal{H}_{B} .
$$

This matrix $M(|e\rangle)$ is positive semidefinite if and only if its diagonal entries and determinant are greater than or equal to zero. From condition 1 it follows that $M_{11} \geqslant 0$ and $M_{22} \geqslant 0$. From conditions 2 and 3,

$$
\begin{aligned}
& \left\langle e\left|\frac{W_{k k}}{d_{A}-1}\right| e\right\rangle\left\langle e\left|\frac{W_{j j}}{d_{A}-1}\right| e\right\rangle \\
& \quad \geqslant 2\left[\max \left\{\left\langle e\left|\frac{\sqrt{2}}{2}\left(W_{k j}+W_{j k}\right)\right| e\right\rangle,\left\langle e\left|\frac{\sqrt{2}}{2 i}\left(W_{k j}-W_{j k}\right)\right| e\right\rangle\right\}\right]^{2} \\
& \geqslant\left\langle e\left|W_{k j}\right| e\right\rangle\left\langle e\left|W_{j k}\right| e\right\rangle \\
& \Rightarrow \operatorname{det}(M(|e\rangle)>0 . \text { QED. }
\end{aligned}
$$

We present now the first example of our methodology. We used MATLAB and the package SEDUMI [11] to implement and solve the SDP.

\section{A. Bell state}

We consider the Bell state $|\Psi\rangle=(|0\rangle \otimes|0\rangle+|1\rangle \otimes|1\rangle) / \sqrt{2}$. It is well known that $\left\langle\Psi\left|W_{|\Psi\rangle\langle\Psi|}\right| \Psi\right\rangle=-1 / 2$ [15], where $W_{|\Psi\rangle\langle\Psi|}=-\frac{1}{2}(|00\rangle\langle 11|+| 11\rangle\langle 00|)+\frac{1}{2}(|01\rangle\langle 01|+| 10\rangle\langle 10|)$ is the OEW for this state. Solving the SDP of Theorem 2, the following EW was found:

$$
W=\left[\begin{array}{cccc}
0.1057 & 0 & 0 & -0.2887 \\
0 & 0.3943 & 0 & 0 \\
0 & 0 & 0.3943 & 0 \\
-0.2887 & 0 & 0 & 0.1057
\end{array}\right] .
$$

Since program (6) is only a relaxation of (5), $W$ is not the OEW for $|\Psi\rangle,\left\langle\Psi\left|W_{\text {opt }}\right| \Psi\right\rangle=-0.1835$.

\section{B. Isospectral states}

We now consider the two isospectral matrices

$$
\rho_{A B}=\left[\begin{array}{cccc}
1 / 3 & 0 & 0 & 0 \\
0 & 1 / 3 & 1 / 3 & 0 \\
0 & 1 / 3 & 1 / 3 & 0 \\
0 & 0 & 0 & 0
\end{array}\right]
$$

$$
\sigma_{A B}=\left[\begin{array}{cccc}
1 / 3 & 0 & 0 & 0 \\
0 & 0 & 0 & 0 \\
0 & 0 & 0 & 0 \\
0 & 0 & 0 & 2 / 3
\end{array}\right]
$$

The positive partial transpose criterion shows that $\rho_{A B}$ is entangled, while $\sigma_{A B}$ is separable. Using the SDP (6), we have found the following $\mathrm{EW}$ for $\rho_{A B}$ :

$$
W=\left[\begin{array}{cccc}
0.1752 & 0 & 0 & 0 \\
0 & 0.1752 & -0.2478 & 0 \\
0 & -0.2478 & 0.0513 & 0 \\
0 & 0 & 0 & 0.5982
\end{array}\right] \text {, }
$$

where $\operatorname{Tr}\left(W \rho_{A B}\right)=-0.0313$. The method has also succeeded in the state $\sigma_{A B}$, as the optimal value for $\operatorname{Tr}\left(W \sigma_{A B}\right)$ found was $2.7330 \times 10^{-5}$.

\section{COMPLETE FAMILY OF PARAMETRIZED RELAXATIONS}

It must be stressed that Theorem 2 is only one of the possible approximations of (5). In fact, every relaxation of the RSDP constitutes a different method of EW construction and, therefore, a new sufficient criterion of separability. In this section we will show that all these possible relaxations can be parametrized in terms of a family of matrices. However, in order to provide such a method, it is necessary first to introduce some standard results concerning robust semidefinite programs. One particularly important representation of robust linear matrix inequalities is the linear fraction representation (LFR) $[13,16]$. It was shown that every matrix $F \in \mathcal{C}^{n \times c}$ which depends rationally on a varying parameter $\delta \in \mathcal{C}^{k}$ can be expressed as [16]

$$
F(\delta)=A+B \Delta(I-D \Delta)^{-1} C,
$$

where $A \in \mathcal{C}^{n \times c}, B \in \mathcal{C}^{n \times N}, C \in \mathcal{C}^{N \times c}$, and $D \in \mathcal{C}^{N \times N}$ are constant matrices, $r_{1}, \ldots, r_{k}$ and $N=r_{1}+\cdots+r_{k}$ are integer numbers, and $\Delta$ is the following diagonal matrix:

$$
\Delta=\operatorname{diag}\left(\delta_{1} I_{r_{1}}, \ldots, \delta_{k} I_{r_{k}}\right) .
$$

We can now express problem (5) in terms of a LFR.

Theorem 3. A state $\rho_{A B}$ is entangled and the optimal value of $W$ is the OEW for it if and only if the result of the following RSDP is negative:

$$
\text { minimize } \operatorname{Tr}\left(W \rho_{A B}\right) \text { subject to }
$$

$$
F(\Delta)=B \Delta(I-D \Delta)^{-1} C>0, \quad \forall \Delta \in \mathcal{D},
$$

where

$$
\begin{gathered}
B=\left[W_{11} \cdots W_{n 1} W_{12} \cdots W_{n n} 0_{d_{A} ; d_{A} d_{B}}\right], \\
C=\left[\begin{array}{ll}
0_{d_{A} ; d_{B}^{2} d_{A}} & L \otimes I_{d_{A}}
\end{array}\right]^{\dagger}, \\
D=\left[\begin{array}{cc}
0_{d_{A} d_{B}^{2} ; d_{A} d_{B}^{2}} & L^{\dagger} \otimes I_{d_{A} d_{B}} \\
0_{d_{A} d_{B} ; d_{A} d_{B}^{2}} & 0_{d_{A} d_{B} ; d_{A} d_{B}}
\end{array}\right],
\end{gathered}
$$




$$
\Delta=\operatorname{diag}\left(a_{1} I_{d_{A} d_{B}}, \ldots, a_{d_{A}} I_{d_{A} d_{B}}, a_{1}^{*} I_{d_{A}}, \ldots, a_{d_{A}}^{*} I_{d_{A}}\right),
$$

and $\mathcal{D}$ is the subspace of diagonal matrices in the form of (12) where $a_{j} \in \mathcal{C}$. $0_{p ; q}$ and $I_{p}$ stand for the $p \times q$ zero matrix and the $p \times p$ identity matrix, respectively. $L$ is an auxiliary matrix given by

$$
L=[1,1, \ldots, 1] \in H_{B} .
$$

Proof. We must show that the LMI of (8) is equivalent to the LMI of (5). In order to do that, we will use constructive formulas of addition and multiplication of LFRs presented in the Appendix. Each quadratic term from the LMI of (5) can be written as

$$
\begin{aligned}
a_{i}^{*} a_{j} W_{i j}= & \left\{0+1 \times a_{i}^{*}\left(1-0 \times a_{i}^{*}\right)^{-1} \times 1\right\} \\
& \times\left\{0+W_{i j} \times a_{i}^{*}\left(I-0 \times a_{i}^{*}\right)^{-1} \times I\right\} .
\end{aligned}
$$

A LFR to each term and then to the whole expression can now be obtained using the addition and the multiplication formulas, respectively. QED.

There are several approximations for robust linear matrix inequalities (RLMIs) which are described as LFRs [13]. One of particular importance is the full block $S$ procedure [17].

Theorem 4 (full block $S$ procedure [17]). The matrix $F(\Delta)=A+B \Delta(I-D \Delta)^{-1} C$ is well posed and satisfies

$$
\left[\begin{array}{c}
I \\
F(\Delta)
\end{array}\right]^{\dagger}\left[\begin{array}{ll}
0 & X \\
X & 0
\end{array}\right]\left[\begin{array}{c}
I \\
F(\Delta)
\end{array}\right] \leqslant 0, \quad \forall \Delta \in \mathcal{D},
$$

if and only if there exists a multiplier

$$
P=\left[\begin{array}{cc}
Q & S \\
S^{\dagger} & R
\end{array}\right]
$$

with

$$
\left[\begin{array}{l}
\Delta \\
I
\end{array}\right]^{\dagger} P\left[\begin{array}{l}
\Delta \\
I
\end{array}\right] \geqslant 0, \quad \forall \Delta \in \mathcal{D},
$$

such that

$$
\left[\begin{array}{cc}
I & 0 \\
A & B \\
0 & I \\
C & D
\end{array}\right]+\left[\begin{array}{cccc}
0 & X & 0 & 0 \\
X & 0 & 0 & 0 \\
0 & 0 & Q & S \\
0 & 0 & S^{\dagger} & R
\end{array}\right]\left[\begin{array}{cc}
I & 0 \\
A & B \\
0 & I \\
C & D
\end{array}\right] \leqslant 0 .
$$

We can now express all possible relaxations of (5) in terms of the multiplier matrix $P$ as follows.

Theorem 5. A state $\rho_{A B}$ is entangled if and only if there exists a multiplier matrix (15) such that (16) and (17) hold, with $X=-I$. The matrices appearing in the LMI (17) are given by Eqs. (10)-(12) and the matrix $\Delta$ is given by (13).

Proof. Noticing that $F(\delta) \geqslant 0$ is equivalent to (14) if $X$ $=-I$, the result follows easily from the application of the full block $S$ procedure (Theorem 4) in the RSDP (9). QED.

The families of matrices $P$ such that (16) is satisfied parametrize all possible relaxations of (5). Although the determination of all such matrices is not a trivial problem, it is a lot easier than (14) and it is the current subject of intensive research. Further information on possible choices of the matrix $P$ can be found in [17]. As an example, we consider now the simplest family of matrices $P$ for which (16) holds. In quantum mechanics one usually deals with normalized states $\langle\psi \mid \psi\rangle=1$. Therefore, the matrix $\Delta$ satisfies $\Delta^{\dagger} \Delta<I$. In this case, the following matrix $P$ gives an approximation of (5):

$$
P=\left[\begin{array}{cc}
-I & 0 \\
0 & I
\end{array}\right]
$$

\section{MULTIPARTITE ENTANGLEMENT}

So far we have only considered the bipartite case. In this section we generalize the previous results to multipartite states. A density operator $\rho_{1, \ldots, n} \in \mathcal{B}\left(H_{1} \otimes \cdots \otimes H_{n}\right)$ is separable if it can be decomposed as

$$
\rho_{1, \ldots, n}=\sum_{i} p_{i}\left|\psi_{i}\right\rangle_{1}\left\langle\psi_{i}|\otimes \cdots \otimes| \psi_{i}\right\rangle_{n}\left\langle\psi_{i}\right|
$$

Since the set of multipartite mixed separable states is also convex, it is possible to apply the Hahn-Banach theorem and establish the concept of the EW in a straightforward manner [18].

Theorem 6. A state $\rho_{1, \ldots, n} \in \mathcal{B}\left(H_{1} \otimes \cdots \otimes H_{n}\right)$ is entangled, i.e., cannot be decomposed as (18) if and only if the optimal value of the following RSDP is negative:

$$
\text { minimize } \operatorname{Tr}\left(W \rho_{1, \ldots, n}\right) \text { subject to }
$$

$$
\begin{gathered}
\sum_{i_{1}=1}^{d_{n}} \sum_{j_{1}=1}^{d_{n}} \cdots \sum_{i_{n-1}=1}^{d_{n}} \sum_{j_{n-1}=1}^{d_{n}}\left(a_{i_{1}}^{*} \cdots a_{i_{n-1}}^{*} a_{j_{1}} \cdots a_{j_{n-1}}\right. \\
\left.\times W_{i_{1}, \ldots, i_{n-1} j_{1}, \ldots, j_{n-1}}\right) \geqslant 0,
\end{gathered}
$$

$$
\operatorname{Tr}(W)=1, \quad \forall a_{i_{k}} \in \mathcal{C}, \quad 1 \leqslant k \leqslant n,
$$

where $d_{n}$ is the dimension of $H_{n}, W_{i_{1}, \ldots, i_{n-1} j_{1}, \ldots, j_{n-1}}={ }_{1}\langle i|$ $\otimes \cdots \otimes_{n-1}\langle i|W| j\rangle_{n-1} \otimes \cdots \otimes|j\rangle_{1} \in \mathcal{B}\left(H_{1} \otimes \cdots \otimes H_{n-1}\right)$ and $|j\rangle_{k}$ is an orthonormal base of $\mathcal{H}_{k}$. If $\rho_{1, \ldots, n}$ is entangled, the matrix $W$ which minimizes $\operatorname{Tr}\left(W \rho_{1, \ldots, n}\right)$ is the OEW for $\rho_{1, \ldots, n}$.

Proof. We know that a state $\rho_{A B}$ is entangled if and only if there exists an operator $W$ such that $\operatorname{Tr}\left(W \rho_{1, \ldots, n}\right) \leqslant 0$ and ${ }_{1}\left\langle\psi\left|\otimes \cdots \otimes{ }_{n}\langle\psi|W| \psi\rangle_{n} \otimes \cdots \otimes\right| \psi\right\rangle_{1} \geqslant 0$ for all states $|\psi\rangle_{k}$ $\in H_{K}$. Thus, the matrix ${ }_{1}\langle\psi| \otimes \cdots \otimes_{n-1}\langle\psi|W| \psi\rangle_{n-1} \otimes \cdots$ $\otimes|\psi\rangle_{1} \geqslant 0$ has to be semidefinite positive for all $|\psi\rangle_{k} \in H_{K}$. Letting $|\psi\rangle_{k}=\sum_{j} a_{j}^{k}|j\rangle_{k}$, where $|j\rangle_{k}$ is an orthonormal base of $\mathcal{H}_{k}$, it is straightforward to show that the optimal $W$ given by (19) is the OEW of $\rho_{1, \ldots, n}$. QED.

Relaxations for (19) can be obtained using the same arguments exposed before. Since the RLMI of (19) is polynomial in the varying parameters, it can be expressed as a LFR and we can apply the full block $S$ procedure to the multipartite case. Therefore, all possible deterministic approximations of (19) can also be parametrized by the matrix $P$. Further results concerning the application of possible families of parametrizations of $P$ in the optimization of the EW will be reported elsewhere. 


\section{CONCLUSION}

In this paper we have introduced, in the context of quantum information, a class of optimization programs (RSDPs) showing that the determination of the OEW for an arbitrary state is $N P$-hard. Several possible deterministic approximation scenarios have been proposed to it, yielding sufficient criteria of separability. Our results were then straightforwardly generalized to multipartite states. It was also shown that all sufficient criteria of separability might be parametrized by a matrix which satisfies a much simpler linear matrix inequality. Therefore, a systematic study of all possible families of parametrizations for this matrix is of great importance.

\section{ACKNOWLEDGMENTS}

Financial support from the Brazilian agencies CNPq, Institutos do Milênio-Informação Quântica, and FAPEMIG is acknowledged.

\section{APPENDIX: COMBINATION OF LFRS}

We provide in this appendix some simple combination rules for addition and multiplication of LFRs used in this paper. Consider two matrices described by the LFR format

$$
F_{i}(\delta)=A_{i}+B_{i} \Delta_{i}\left(I-D_{i} \Delta_{i}\right)^{-1} C_{i} .
$$

The sum of $F_{1}$ and $F_{2}$ has the LFR

$$
F(\delta)=A+B \Delta(I-D \Delta)^{-1} C
$$

with

$$
\begin{gathered}
A=A_{1}+A_{2}, \quad B=\left[\begin{array}{ll}
B_{1} & B_{2}
\end{array}\right], \quad C=\left[\begin{array}{ll}
C_{1} & C_{2}
\end{array}\right]^{\dagger}, \\
D=\operatorname{diag}\left(D_{1}, D_{2}\right), \quad \Delta=\operatorname{diag}\left(\Delta_{1}, \Delta_{2}\right) .
\end{gathered}
$$

The product of $F_{1}$ and $F_{2}$ is given by Eq. (A1) with

$$
\begin{gathered}
A=A_{1} A_{2}, \quad B=\left[B_{1} A_{1} B_{2}\right], \quad C=\left[C_{1} A_{2} C_{2}\right]^{\dagger} \\
D=\left[\begin{array}{cc}
D_{1} & C_{1} B_{2} \\
0 & D_{2}
\end{array}\right], \quad \Delta=\operatorname{diag}\left(\Delta_{1}, \Delta_{2}\right) .
\end{gathered}
$$

[1] A. Einstein, B. Podolsky, and N. Rosen, Phys. Rev. 47, 777 (1935).

[2] M. A. Nielsen and I. L. Chuang, Quantum Computation and Quantum Information (Cambridge University Press, Cambridge, U.K., 2000).

[3] A. Peres, Phys. Rev. Lett. 77, 1413 (1996); M. Lewenstein, B. Kraus, J. I. Cirac, and P. Horodecki, e-print quant-ph/0005112; M. Horodecki and P. Horodecki, Phys. Rev. A 59, 4206 (1999); B. M. Terhal, e-print quant-ph/9911057.

[4] L. Gurvits, in Proceedings of the 35th ACM Symposium on the Theory of Computing (ACM Press, New York, 2003), pp. 1019.

[5] M. Horodecki, P. Horodecki, and R. Horodecki, Phys. Lett. A 223, 8 (1996).

[6] B. M. Terhal, e-print quant-ph/9810091.

[7] M. Lewenstein, B. Kraus, J. I. Cirac, and P. Horodecki, e-print quant-ph/0005014.

[8] Y. Nesterov and A. Nemirovskii, Interior Point Polynomial Methods in Convex Programming: Theory and Applications, Studies in Applied Mathematics Series (SIAM, Philadelphia, PA, 1994).

[9] Y. C. Eldar, IEEE Trans. Inf. Theory 49, 446 (2003); M. Jezek,
J. Rehacek, and J. Fiurasek, Phys. Rev. A 65, 060301 (2002).

[10] A. C. Doherty, P. A. Parrilo, and F. M. Spedalieri, Phys. Rev. A 69, 022308 (2004).

[11] See, for example, SEDUMI, http://fewcal.kub.nl/sturm/software/ sedumi.html

[12] A. Ben Tal and A. Nemirovski, Math. Op. Res. 23, 769 (1998).

[13] P. Apkarian and H. D. Tuan, SIAM J. Control Optim. 38, 1241 (2000); L. El Ghaoui and H. Lebret, SIAM J. Matrix Anal. Appl. 18, 1035 (1997).

[14] H. D. Tuan, P. Apkarian, T. Narikiyo, and Y. Yamamoto, IEEE Trans. Fuzzy Syst. 9, 324 (2001).

[15] O. Guehne, P. Hyllus, D. Bruss, A. Ekert, M. Lewenstein, C. Macchiavello, and A. Sanpera, Phys. Rev. A 66, 062305 (2002).

[16] K. Zhou, J. Doyle, and K. Glover, Robust and Optimal Control (Prentice-Hall, Upper Saddle River, NJ, 1995).

[17] C. W. Scherer, in Proceedings of IEEE Conference on Decision and Control, San Diego, 1997 (unpublished), pp. 26022607.

[18] M. Horodecki, P. Horodecki, and R. Horodecki, e-print quant$\mathrm{ph} / 0006071$. 